\title{
On the Asymptotic Limit of the Three Dimensional Vlasov-Poisson System for Large Magnetic Field: Formal Derivation
}

\author{
Pierre Degond $^{1}$ (D) Francis Filbet ${ }^{2}$
}

Received: 10 March 2016/ Accepted: 18 October 2016/ Published online: 1 November 2016

(C) The Author(s) 2016. This article is published with open access at Springerlink.com

\begin{abstract}
In this paper we establish the asymptotic limit of the three dimensional VlasovPoisson equation with strong external magnetic field. The guiding center approximation is investigated in the three dimensional case with a non-constant magnetic field. In the long time asymptotic limit, the motion can be split in two parts: one stationary flow along the lines of the magnetic field and the guiding center motion in the orthogonal plane of the magnetic field where classical drift velocities and invariants (magnetic moment) are recovered.
\end{abstract}

Keywords Vlasov-Poisson system · Guiding-centre model · Asymptotic expansion

Mathematics Subject Classification 78A35 - 35Q60 - 82D10

\section{Introduction}

We consider a plasma confined by a strong external non constant magnetic field, hence the charged gas evolves under its self-consistent electrostatic field and the confining magnetic field. This configuration is typical of a tokamak plasma [3,39] where the magnetic field is used to confine particles inside the core of the device.

We assume that on the time scale we consider, collisions can be neglected both for ions and electrons, hence collective effects are dominant and the plasma is entirely modelled with kinetic transport equations, where the unknown is the number density of particles $f \equiv$ $f(t, \mathbf{x}, \mathbf{v})$ depending on time $t \geq 0$, position $\mathbf{x} \in \Omega \subset \mathbb{R}^{3}$ and velocity $\mathbf{v} \in \mathbb{R}^{3}$.

\section{Pierre Degond}

pdegond@imperial.ac.uk

\section{Francis Filbet}

francis.filbet@math.univ-toulouse.fr

1 Department of Mathematics, Imperial College of London, London SW7 2AZ, UK

2 Institut de Mathématiques de Toulouse, Université Toulouse III, Institut Universitaire de France, 118, route de Narbonne, 31062 Toulouse cedex, France 
Such a kinetic model provides an appropriate description of turbulent transport in a fairly general context, but it requires to solve a six dimensional problem which leads to a huge computational cost.

To reduce the cost of numerical simulations, it is classical to derive asymptotic models with a smaller number of variables than the kinetic description. Large magnetic fields usually lead to the so-called drift-kinetic limit $[1,14,31,32]$ and we refer to $[9,13,18-20,23]$ for recent mathematical results on this topic. In this regime, due to the large applied magnetic field, particles are confined along the magnetic field lines and their period of rotation around these lines (called the cyclotron period) becomes small. It corresponds to the finite Larmor radius scaling for the Vlasov-Poisson equation, which was introduced by Frénod and Sonnendrücker in the mathematical literature $[18,19]$. The two-dimensional version of the system (obtained when one restricts to the perpendicular dynamics) and the large magnetic field limit were studied in [20] and more recently in [9,22,26]. We also refer to the recent work [30] of Hauray and Nouri, dealing with the well-posedness theory with a diffusive version of a related two dimensional system. A version of the full three dimensional system describing ions with massless electrons was studied by Han-Kwan in [27,29]. In the context of space plasmas, C. Cheverry also recently proposed a study of the Vlasov-Maxwell system with a strong external magnetic field [4] in the long time asymptotic to justify the validity of gyro-kinetic models and achieved a precise analysis of oscillatory integrals to understand the confinement property of the external magnetic field.

Here, we formally derive a new asymptotic model under both assumptions of large magnetic fields and large time asymptotic limit for the three dimensional Vlasov-Poisson system. Analogous problem has already been carefully studied by F. Golse and L. Saint-Raymond in two dimension $[23,24,42]$.

We consider a plasma model in which we focus on the dynamics of the fast electrons and the magnetic field is assumed to be given. In the large magnetic field regime, the Lorentz force term in the Vlasov equation is scaled by a large parameter, $1 / \varepsilon$, where $\varepsilon$ stands for the dimensionless ion cyclotron period, i.e. the rotation period of the electrons about a magnetic field line (or Larmor rotation). The so called drift-kinetic or gyro-kinetic regimes are reached when $\varepsilon$ tends to zero (see $[31,36]$ ).

Here we are interested in the long time behavior of the distribution of electrons since they can be considered as fast particles compared to the characteristic velocity of ions. In this limit, the new distribution function only depends on space, time and two components of the velocity, corresponding to the parallel component along the magnetic field line and the magnitude of the perpendicular velocity. In other words, the distribution function is independent of the gyro-phase of the perpendicular velocity in the plane normal to the magnetic field line. This is a consequence of the ultra-fast cyclotron rotation about the magnetic field lines.

It is also convenient to express the distribution in terms of the parallel velocity and the magnetic moment or adiabatic invariant, which is proportional to the perpendicular energy divided by the magnitude of the magnetic field.

The present work is an attempt to give a mathematical framework of the theory developed for plasma confinement in theoretical physics [32]. Using our approach, the distribution function in these new variables satisfies a transport equation with a constraint. A Lagrange multiplier allows to express this constraint in the differential system. More precisely, the constraint comes from the perturbation and imposes that the distribution function is constant along the trajectories of the fast parallel motion along the magnetic field lines. The resulting asymptotic model seems to be different to the models in [32] but these differences only come from a rigorous treatment of the terms which have been neglected in [32]. However, our asymptotic model shares some basic properties with classical ones (drift kinetic model, guid- 
ing center equation) since we recover drift velocity, energy conservation, magnetic moment invariant.

The derivation of the model roughly follows the following steps: we first proceed with formal expansions of the distribution function with respect to the parameter $\varepsilon$. Now, carrying the Hilbert expansion procedure through for the distribution function equation is best done if we change the random velocity variable into a coordinate system consisting of the parallel velocity, the energy, and the angle of rotation or gyrophase around the magnetic field line.

Thanks to this coordinate change, we show that the leading order term of the distribution function does not depend on the gyrophase. Next, we realize that, at each level of the expansion, we are led to inverting the gyrophase averaging operator [31,32]. We show that the inverse operator can only act on functions satisfying a specific solvability condition, namely that their gyrophase average is zero. We find the asymptotic model following the classical Hilbert expansion procedure of kinetic theory. Providing an explicit expression of the Hilbert expansion procedure is achieved here assuming that the magnetic field only acts in the $z$ direction.

The remainder of the paper is organized as follows. In Sect. 2, we present the scaling which expresses the assumptions of strong magnetic field and long time asymptotic regime. Then, we present and comment the main result of this article, namely the asymptotic model. In Sect. 4, by using Hilbert expansions we derive the asymptotic model and provide the main computational steps which lead to the explicit partial differential system for the limit distribution function. Finally, in Sect. 5 we give several directions for future work (description of the curvature effects, existence theory of the asymptotic model, numerical simulations).

\section{Scaling and Main Results}

\subsection{The Vlasov Equation in a Strong Magnetic Field}

We are interested in the fast dynamics of the negatively charged electrons in the plasma. At this stage of the study, the coupling with the ions is discarded and the electric field is given by the Poisson equation whereas the magnetic field is external.

We investigate the asymptotic limit of the Vlasov equation describing the long time dynamics of the electrons when they are submitted to an asymptotically large external magnetic field.

Denoting by $m$ the electron mass and by $q$ the negative charge of the electron, we start from the Vlasov equation

$$
\frac{\partial f}{\partial t}+\mathbf{v} \cdot \nabla_{\mathbf{x}} f+\frac{q}{m}\left(\mathbf{E}+\mathbf{v} \times \mathbf{B}_{\mathrm{ext}}\right) \cdot \nabla_{\mathbf{v}} f=0
$$

where $f \equiv f(t, \mathbf{x}, \mathbf{v})$ is the distribution function and $\mathbf{x} \in \Omega \subset \mathbb{R}^{3}, \mathbf{v} \in \mathbb{R}^{3}$, and $t \in \mathbb{R}^{+}$ are respectively the position, velocity, and time variables. Also, $\mathbf{B}_{\text {ext }}$ is the external magnetic field applied to confine the charged particles, whereas $\mathbf{E}$ represents the self-consistent electric field such that $\mathbf{E}=\nabla \phi$ and the potential $\phi$ is solution to the Poisson equation

$$
-\Delta \phi=\frac{4 \pi}{\epsilon_{0}}\left(\rho-\rho_{0}\right),
$$


where $\epsilon_{0}$ is the permittivity of the vacuum and $\rho_{0}$ represents the given ion density and $\rho$ is the charge density of electrons

$$
\rho=q \int_{\mathbb{R}^{3}} f d \mathbf{v}
$$

Then, we prescribe an initial datum

$$
f(0, \mathbf{x}, \mathbf{v})=f_{\text {in }}(\mathbf{x}, \mathbf{v}), \quad \mathbf{x} \in \Omega, \quad \mathbf{v} \in \mathbb{R}^{3},
$$

where $f_{\text {in }}$ is the distribution function of particles initially present inside the domain $\Omega$.

Next, we introduce a set of characteristic scales from which an appropriate scaling of equation (1) will be derived.

The characteristic length scale of the problem $\bar{x}$ is the Debye length

$$
\lambda_{D}=\left(\frac{k_{\mathcal{B}} \epsilon_{0} \bar{T}}{4 \pi \bar{n} q^{2}}\right)^{1 / 2},
$$

where $k_{\mathcal{B}}$ is the Boltzmann constant, $\bar{T}$ is the temperature scale and $\bar{n}$ is the density scale. Then, the characteristic magnitude of the electric field can be expressed from $\bar{n}$ and $\bar{x}$ by $\bar{E}=4 \pi q \bar{n} \bar{x} / \epsilon_{0}$ and the characteristic velocity of electrons $\bar{v}$ is the thermal velocity of the electrons, $v_{t h}=\left(k_{\mathcal{B}} \bar{T} / m\right)^{1 / 2}$.

Therefore, the plasma frequency of electrons satisfies

$$
\omega_{p}^{-1}=\frac{\bar{x}}{\bar{v}}
$$

which corresponds to one time scale. Moreover, we denote by $\bar{B}$ the characteristic magnitude of the applied magnetic field and define $\omega_{c}=\frac{q \bar{B}}{m}$ the characteristic electron cyclotron frequency, and $\omega_{c}^{-1}$ corresponds to a second time scale.

Hence we define the new variables and given fields by

$$
\mathbf{x}^{\prime}=\frac{\mathbf{x}}{\bar{x}}, \quad \mathbf{v}^{\prime}=\frac{\mathbf{v}}{\bar{v}}, \quad t^{\prime}=\frac{t}{\bar{t}}, \quad \mathbf{E}^{\prime}\left(t^{\prime}, \mathbf{x}^{\prime}\right)=\frac{\mathbf{E}(t, \mathbf{x})}{\bar{E}}, \quad \mathbf{B}_{\mathrm{ext}}^{\prime}\left(t^{\prime}, \mathbf{x}^{\prime}\right)=\frac{\mathbf{B}_{\mathrm{ext}}(t, \mathbf{x})}{\bar{B}} .
$$

Subsequently, letting $\bar{f}=\bar{n} / \bar{v}^{3}$ the distribution function scale, we introduce the new unknown

$$
f^{\prime}\left(t^{\prime}, \mathbf{x}^{\prime}, \mathbf{v}^{\prime}\right)=\frac{f(t, \mathbf{x}, \mathbf{v})}{\bar{f}} .
$$

Inserting all these changes into (1), dividing by $\omega_{p}$ and dropping the primes for clarity, we obtain the dimensionless equation

$$
\frac{1}{\omega_{p} \bar{t}} \frac{\partial f}{\partial t}+\mathbf{v} \cdot \nabla_{\mathbf{x}} f+\left(\mathbf{E}+\frac{\omega_{c}}{\omega_{p}} \mathbf{v} \times \mathbf{B}_{\mathrm{ext}}\right) \cdot \nabla_{\mathbf{v}} f=0
$$

When the external magnetic field is assumed to be large, the rotation period of the electrons about the magnetic field lines becomes small. We introduce the dimensionless cyclotron period

$$
\varepsilon=\frac{\omega_{p}}{\omega_{c}}
$$


and since we are interested in asymptotically large time scale, we also have that

$$
\varepsilon=\frac{1}{\bar{t} \omega_{p}} .
$$

Then, under this scaling, the Vlasov equation (3) for $f=f_{\varepsilon}$ takes the form:

$$
\varepsilon \frac{\partial f^{\varepsilon}}{\partial t}+\mathbf{v} \cdot \nabla_{\mathbf{x}} f^{\varepsilon}+\left(\mathbf{E}^{\varepsilon}+\frac{1}{\varepsilon} \mathbf{v} \times \mathbf{B}_{\mathrm{ext}}\right) \cdot \nabla_{\mathbf{v}} f^{\varepsilon}=0,
$$

with the Poisson equation for the potential, $\phi$ such that $\mathbf{E}^{\varepsilon}=-\nabla \phi^{\varepsilon}$ and

$$
-\Delta \phi^{\varepsilon}=\int_{\mathbb{R}^{3}} f^{\varepsilon} d \mathbf{v}-\rho_{0},
$$

and initial conditions still given by (2).

\subsection{Assumptions and Main Result}

To simplify the presentation and the following calculation, we assume that the domain is $\Omega:=Q \times\left(0, L_{z}\right)$, with $Q \subset \mathbb{R}^{2}$ and the external magnetic field only applies in the $z-$ direction

$$
\mathbf{B}_{\text {ext }}(t, \mathbf{x})=\left(0,0, b\left(t, \mathbf{x}_{\perp}\right)\right)^{t},
$$

where $\mathbf{x}=(x, y, z)^{t} \in \Omega$ with $\mathbf{x}_{\perp}=(x, y)$ and $x_{\|}=z$. The velocity variable will be denoted in the same manner $\mathbf{v}=\left(\mathbf{v}_{\perp}, v_{\|}\right)$, with $\mathbf{v}_{\perp}=\left(v_{x}, v_{y}\right)$ and $v_{\|}=v_{z}$.

Since the external magnetic field must satisfy the Gauss's law for magnetism

$$
\nabla_{\mathbf{x}} \cdot \mathbf{B}_{\mathrm{ext}}=0,
$$

it gives that indeed $b$ only depends on $x_{\perp} \in \mathbb{R}^{2}$ and $t \in \mathbb{R}^{+}$. Furthermore, we assume that $b$ does not vanish and is smooth: there exists $\alpha>0$ such that

$$
b \in W^{1, \infty}\left(\mathbb{R}^{+} \times Q\right), \quad b\left(t, \mathbf{x}_{\perp}\right)>\alpha .
$$

A natural assumption is that the distribution function is periodic in the $z$-direction

$$
f^{\varepsilon}(t, \mathbf{x}, \mathbf{v})=f^{\varepsilon}\left(t, \mathbf{x}+L_{z} \mathbf{e}_{z}, \mathbf{v}\right), \quad(\mathbf{x}, \mathbf{v}) \in \Omega \times \mathbb{R}^{3},
$$

but for simplicity of the presentation, we also assume either periodic boundary condition in the domain $Q$ or $Q=\mathbb{R}^{2}$. Hence, we have the compatibility condition

$$
\int_{\Omega} \rho^{\varepsilon}(t, \mathbf{x}) d \mathbf{x}=\int_{\mathbb{R}^{3} \times \Omega} f^{\varepsilon}(t, \mathbf{x}, \mathbf{v}) d \mathbf{v} d \mathbf{x}=\int_{\Omega} \rho_{0}(\mathbf{x}) d \mathbf{x} .
$$

Under these assumptions, the Vlasov equation (4) can be written in a simple form, which allows us to ignore curvature effects

$$
\left\{\begin{array}{l}
\varepsilon \frac{\partial f^{\varepsilon}}{\partial t}+\mathbf{v} \cdot \nabla_{\mathbf{x}} f^{\varepsilon}+\left(\mathbf{E}^{\varepsilon}+\frac{b}{\varepsilon} \mathbf{v}^{\perp}\right) \cdot \nabla_{\mathbf{v}} f^{\varepsilon}=0, \\
-\Delta \phi^{\varepsilon}=\rho^{\varepsilon}-\rho_{0}=\int_{\mathbb{R}^{3}} f^{\varepsilon} d \mathbf{v}-\rho_{0}, \quad \mathbf{E}^{\varepsilon}=-\nabla_{\mathbf{x}} \phi^{\varepsilon}, \\
f^{\varepsilon}(0)=f_{\text {in }}^{\varepsilon},
\end{array}\right.
$$

where the operator $\mathbf{v}^{\perp}=\left(v_{y},-v_{x}, 0\right)$ and therefore it only acts on the $\mathbf{v}_{\perp}=\left(v_{x}, v_{y}\right)$ component and keeps the third component identical : for any $\mathbf{v}=\left(\mathbf{v}_{\perp}, v_{\|}\right) \in \mathbb{R}^{3}$, we have $\mathbf{v}^{\perp}=\left(v_{y},-v_{x}, 0\right)$. 
Let us first emphasize that applying the arguments of A.A. Arsen'ev [2] and R. DiPerna and P.-L. Lions [6], we easily prove the existence of weak solutions for any $\varepsilon>0$.

Theorem 2.1 Assume the magnetic field satisfies (5)-(6) and the initial datum $f_{\text {in }}^{\varepsilon}$ is a nonnegative function such that

$$
f_{\text {in }}^{\varepsilon} \in L^{1} \cap L^{\infty}\left(\Omega \times \mathbb{R}^{3}\right)
$$

and has finite kinetic energy

$$
\frac{1}{2} \int_{\Omega \times \mathbb{R}^{3}}|\mathbf{v}|^{2} f_{\text {in }}^{\varepsilon} d \mathbf{x} d \mathbf{v}<\infty
$$

with the compatibility condition (8). Then there is a weak solution $\left(f^{\varepsilon}, \mathbf{E}^{\varepsilon}\right)$ to the VlasovPoisson system (9) with periodic boundary conditions (7), where $f^{\varepsilon} \in L^{\infty}\left(\mathbb{R}^{+}, L^{1} \cap L^{\infty}(\Omega \times\right.$ $\left.\mathbb{R}^{3}\right)$ ), the charge densities $\rho^{\varepsilon}$ is such that

$$
\rho^{\varepsilon} \in L^{\infty}\left(\mathbb{R}^{+} ; L^{5 / 3}(\Omega)\right)
$$

and

$$
\mathbf{E}^{\varepsilon} \in L^{\infty}\left(\mathbb{R}^{+} ; L^{2} \cap W^{1,5 / 3}(\Omega)\right) .
$$

Note here that the $L^{p}$ and energy estimates hold uniformly with respect to $\varepsilon>0$. Furthermore, this results strongly relies on the energy estimate, which is uniform with respect to $\varepsilon>0$. We define the total energy associated to (9)

$$
\mathcal{E}^{\varepsilon}(t):=\int_{\Omega \times \mathbb{R}^{3}} \frac{|\mathbf{v}|^{2}}{2} f^{\varepsilon}(t) d \mathbf{x} d \mathbf{v}+\frac{1}{2} \int_{\Omega}\left|\mathbf{E}^{\varepsilon}\right|^{2} d \mathbf{x} \leq \mathcal{E}^{\varepsilon}(0) .
$$

The aim of this paper is then to obtain a systematic expansion of Hilbert type of the function $\left(f^{\varepsilon}, \mathbf{E}^{\varepsilon}\right)$ solution to the Vlasov-Poisson system (9) and to study the asymptotic model formally obtained by taking the limit $\varepsilon \rightarrow 0$.

Let us assume that $\left(f^{\varepsilon}, \mathbf{E}^{\varepsilon}\right)$ can be written as

$$
f^{\varepsilon}=\sum_{k \in \mathbb{N}} \varepsilon^{k} f_{k}, \quad \mathbf{E}^{\varepsilon}=\sum_{k \in \mathbb{N}} \varepsilon^{k} \mathbf{E}_{k},
$$

where for any $k \in \mathbb{N}, f_{k}$ and $\mathbf{E}_{k}$ do not depend on $\varepsilon$. The existence of such an expansion would guarantee that $f^{\varepsilon}$ and $\mathbf{E}^{\varepsilon}$ and their derivatives with respect to $\mathbf{x}$ and $\mathbf{v}$ are uniformly bounded, at least if the functions $f_{k}, \mathbf{E}_{k}$ are sufficiently smooth.

In particular we assume that

$$
f_{\text {in }}^{\varepsilon}=\sum_{k \in \mathbb{N}} \varepsilon^{k} f_{\text {in }, k},
$$

where for any $k \in \mathbb{N}, f_{\text {in, } k}$ does not depend on $\varepsilon>0$.

To introduce the gyroaveraging operator in the orthogonal plane to magnetic field, we will work in polar coordinate for $\mathbf{v}_{\perp}=\left(v_{x}, v_{y}\right) \in \mathbb{R}^{2}$,

$$
\left\{\begin{array}{l}
v_{x}=w \cos (\theta) \\
v_{y}=w \sin (\theta)
\end{array}\right.
$$

and set $\mathbf{e}_{w}=(\cos \theta, \sin \theta), \mathbf{e}_{\theta}=-\mathbf{e}_{w}^{\perp}=(-\sin \theta, \cos \theta)$. Then we introduce the gyroaveraging operator $\Pi$ defined for every function $f(\mathbf{v})$ by

$$
\Pi f\left(w, v_{\|}\right)=\frac{1}{2 \pi} \int_{0}^{2 \pi} f\left(\mathbf{v}_{\perp}, v_{\|}\right) d \theta,
$$

where in the integral $\mathbf{v}_{\perp}$ is expressed thanks to the change of coordinate (14). 
Theorem 2.2 [Formal expansion of $f^{\varepsilon}$ and $\mathbf{E}^{\varepsilon}$ ] Let us consider an external the magnetic field such that (5)-(6) and $f_{\text {in }}^{\varepsilon}$ a nonnegative function satisfying (10), (11) and (13). Assume there exists a sequence $\left(f_{k}, \mathbf{E}_{k}\right)_{k \in \mathbb{N}}$ such that the weak solutions $\left(f^{\varepsilon}, \mathbf{E}^{\varepsilon}\right)_{\varepsilon}$ to the Vlasov-Poisson system (9) can be expanded as (12) for all $\varepsilon>0$. Then,

$$
\left\{\begin{array}{l}
\mathbf{E}_{0} \equiv \mathbf{E}_{F}(t, \mathbf{x}) \\
f_{0} \equiv F\left(t, \mathbf{x}, w, v_{\|}\right)
\end{array}\right.
$$

and there exists $\left(P, \mathbf{E}_{P}\right)$ with $P \equiv P\left(t, \mathbf{x}, w, v_{\|}\right)$such that $\left(F, P, \mathbf{E}_{F}, \mathbf{E}_{P}\right)$ is a solution to the following system

$$
\left\{\begin{array}{l}
\frac{\partial F}{\partial t}+\mathbf{U}_{\perp} \cdot \nabla_{\mathbf{x}_{\perp}} F+u_{w} \frac{\partial F}{\partial w}-\frac{\partial \phi_{P}}{\partial x_{\|}} \frac{\partial F}{\partial v_{\|}}+v_{\|} \frac{\partial P}{\partial x_{\|}}-\frac{\partial \phi_{F}}{\partial x_{\|}} \frac{\partial P}{\partial v_{\|}}=0 \\
v_{\|} \frac{\partial F}{\partial x_{\|}}-\frac{\partial \phi_{F}}{\partial x_{\|}} \frac{\partial F}{\partial v_{\|}}=0 \\
F(0)=\Pi f_{\text {in }, 0} \\
P(0)=\Pi f_{\text {in, }, 1}
\end{array}\right.
$$

where $\mathbf{U}_{\perp}$ corresponds to the drift velocity and $\left(\mathbf{U}_{\perp}, u_{w}\right)$ is given by

$$
\mathbf{U}_{\perp}=-\frac{1}{b}\left(\nabla_{\mathbf{x}_{\perp}} \phi_{F}+\frac{w^{2}}{2 b} \nabla_{\mathbf{x}_{\perp}} b\right)^{\perp}, \quad u_{w}=\frac{w}{2 b^{2}} \nabla_{\mathbf{x}_{\perp}}^{\perp} b \cdot \nabla_{x_{\perp}} \phi_{F},
$$

and the electric fields $\mathbf{E}_{F}=-\nabla \phi_{F}, \mathbf{E}_{P}=-\nabla \phi_{P}$ are such that $\left(\phi_{F}, \phi_{P}\right)$ solves Poisson equations with source terms $\left(\rho_{F}-\rho_{0}, \rho_{P}\right)$,

$$
\left\{\begin{array}{l}
-\Delta \phi_{F}=2 \pi \int_{\mathbb{R}^{+} \times \mathbb{R}} F\left(t, \mathbf{x}, w, v_{\|}\right) w d w d v_{\|}-\rho_{0}, \\
-\Delta \phi_{P}=2 \pi \int_{\mathbb{R}^{+} \times \mathbb{R}} P\left(t, \mathbf{x}, w, v_{\|}\right) w d w d v_{\|}
\end{array}\right.
$$

with the compatibility condition (8)

$$
2 \pi \int_{\Omega \times \mathbb{R}^{+} \times \mathbb{R}} F w d w d v_{\|} d \mathbf{x}=\int_{\Omega} \rho_{0} d \mathbf{x}, \quad \int_{\Omega \times \mathbb{R}^{+} \times \mathbb{R}} P w d w d v_{\|} d \mathbf{x}=0 .
$$

Moreover, the following relation holds

$$
f_{1}=-\frac{1}{b\left(t, \mathbf{x}_{\perp}\right)} \mathbf{e}_{\theta} \cdot\left(w \nabla_{\mathbf{x}_{\perp}} F+\mathbf{E}_{F \perp} \frac{\partial F}{\partial w}\right)+P\left(t, \mathbf{x}, r, v_{\|}\right) .
$$

Remark 2.3 The transport equation (16) is not standard in kinetic theory due to the presence of the transport operator acting on the pertubation $P$ for the variables $\left(x_{\|}, v_{\|}\right)$. This operator acts as a source term in order to ensure that at any time $t>0$, the following equation

$$
v_{\|} \frac{\partial F}{\partial x_{\|}}-\frac{\partial \phi_{F}}{\partial x_{\|}} \frac{\partial F}{\partial v_{\|}}=0
$$

is satisfied. This results indicates that when we are interested in the asymptotic behavior of the solution $\left(f^{\varepsilon}, \mathbf{E}^{\varepsilon}\right)$ to the Vlasov-Poisson sytem, when $\varepsilon \rightarrow 0$, we cannot ignore the effect of the perturbation corresponding to

$$
\left\{\begin{array}{l}
f^{\varepsilon}=F+\varepsilon f_{1} \\
\mathbf{E}^{\varepsilon}=\mathbf{E}_{F}+\varepsilon \mathbf{E}_{P}
\end{array}\right.
$$

where $f_{1}$ is given by (19). 
Let us emphasize that the first equation of (16) means that $F$ and $P$ do not depend of the gyrophase $\theta \in[0,2 \pi]$, but on $w=\left|\mathbf{v}_{\perp}\right|$. In this model, $F$ is determined by (16) while the unknown function $P$ plays the role of the Lagrange multiplier associated to the constraint in (16). This constraint reflects the fact that the fast parallel motion along the magnetic field line is instantaneously relaxed and $F$ is constant along these trajectories. In other words, there are three time scales for a particle moving in a large magnetic field:

- The fastest time scale corresponds to the cyclotron or Larmor rotation period about the magnetic field. This time scale is eliminated here by averaging over $\theta \in(0,2 \pi)$;

- The second fastest scale is the scale of the parallel motion along the magnetic field line, which is described here by the constraint in (16);

- The slow time scale corresponds to the various drifts across the magnetic field lines, due to spatio-temporal variations of the electromagnetic field. In the system (16), we focus on the slow time scale, which corresponds to the large time behavior of the solution to the Vlasov-Poisson system (9).

Of course these various drifts are often obtained directly on the particle trajectories, but the averaging effect is difficult to justify and is only valid for slowly varying electromagnetic fields. The use of the kinetic model directly provides a way to do it by imposing constraints on the distribution function. This easier derivation reflects the fact that, to some extent, the distribution function describes the particle dynamics in a statistical sense. Averaging the trajectories over some fast component is best done by looking at the evolution of an observable of the system which is constant over this fast motion.

\section{Fundamental Properties of the Asymptotic Model}

In this section, we prove some fundamental properties satisfied by the asymptotic model (16), which illustrates the physical validity of the present approach. In the following, we show that

- We recover the classical drift velocity $\mathbf{E} \times \mathbf{B}$ and the gradient drift velocity;

- The asymptotic model satisfies conservation of energy;

- The magnetic moment is an invariant of the asymptotic model.

\subsection{Drift Velocities}

The drift velocity $\mathbf{U}_{\perp}$ corresponds to the sum of classical guiding center drift $\mathbf{U}_{\mathbf{E}}$ and $\mathbf{U}_{\nabla \mathbf{B}}$. Indeed, the external magnetic field only acts on the $z$-direction, $\mathbf{B}_{\mathrm{ext}}=(0,0, b)$, then we first recover the drift velocity called $\mathbf{E} \times \mathbf{B}$

$$
\mathbf{U}_{\mathbf{E}}=\frac{\mathbf{E} \times \mathbf{B}_{\mathrm{ext}}}{\left\|\mathbf{B}_{\mathrm{ext}}\right\|^{2}}=-\frac{\nabla_{\mathbf{x}_{\perp}}^{\perp} \phi_{F}}{b}
$$

and the so called gradient- $B$ drift,

$$
\mathbf{U}_{\nabla \mathbf{B}}=\frac{\left|\mathbf{v}_{\perp}\right|^{2}}{2} \frac{\mathbf{B}_{\mathrm{ext}} \times \nabla\left|\mathbf{B}_{\mathrm{ext}}\right|}{\left\|\mathbf{B}_{\mathrm{ext}}\right\|^{3}}=-\frac{w^{2}}{2} \frac{\nabla_{\mathbf{x}_{\perp}}^{\perp} b}{b^{2}} .
$$

Here there is no curvature drift since we considered this simple external magnetic field $\mathbf{B}_{\mathrm{ext}}=(0,0, b)$. 


\section{2 $L^{p}$ Norms and Total Energy Conservation}

Let us first write (16) in a conservative form

$$
\begin{aligned}
& \frac{\partial}{\partial t}(w F)+\nabla_{\mathbf{x}_{\perp}} \cdot\left(w \mathbf{U}_{\perp} F\right)+\frac{\partial}{\partial w}\left(w u_{w} F\right)-\frac{\partial}{\partial v_{\|}}\left(\frac{\partial \phi_{P}}{\partial x_{\|}} w F\right) \\
& +\frac{\partial}{\partial x_{\|}}\left(w v_{\|} P\right)-\frac{\partial}{\partial v_{\|}}\left(w \frac{\partial \phi_{F}}{\partial x_{\|}} P\right)=0 .
\end{aligned}
$$

Thus, we prove the following conservation property.

Proposition 3.1 Let $f_{\text {in }}^{\varepsilon}$ be a nonnegative function satisfying (10)-(11) and (13). Assume that the limiting system (16) has a smooth solution $\left(F, \mathbf{E}_{F}\right)$ and $\left(P, \mathbf{E}_{P}\right)$. Then, for any $\Theta \in \mathcal{C}^{1}(\mathbb{R})$ such that

$$
\int_{\Omega \times \mathbb{R}^{+} \times \mathbb{R}} \Theta(F(0)) w d w d v_{\|} d \mathbf{x}<+\infty
$$

we have

$$
\int_{\Omega \times \mathbb{R}^{+} \times \mathbb{R}} \Theta(F(t)) w d w d v_{\|} d \mathbf{x}=\int_{\Omega \times \mathbb{R}^{+} \times \mathbb{R}} \Theta(F(0)) w d w d v_{\|} d \mathbf{x} .
$$

Proof Assuming that $F$ is a smooth solution to (16) together with a smooth $P$, we multiply (16) by $\Theta^{\prime}(F)$ and integrate with respect to $\left(\mathbf{x}, w, v_{\|}\right) \in \Omega \times \mathbb{R}^{+} \times \mathbb{R}$. Then, using that

$$
w \nabla_{\mathbf{x}_{\perp}} \cdot \mathbf{U}_{\perp}=-\frac{w}{b^{2}} \nabla_{\mathbf{x}_{\perp}}^{\perp} b \cdot \nabla_{\mathbf{x}_{\perp}} \phi_{F}
$$

and observing that

$$
v_{\|} \frac{\partial \Theta^{\prime}(F)}{\partial x_{\|}}-\frac{\partial \phi_{F}}{\partial x_{\|}} \frac{\partial \Theta^{\prime}(F)}{\partial v_{\|}}=0,
$$

we easily get after a simple integration by part and for suitable boundary conditions (either periodic or vanishing property in the whole space)

$$
\frac{d}{d t} \int_{\Omega \times \mathbb{R}^{+} \times \mathbb{R}} \Theta(F(t)) w d w d v_{\|} d \mathbf{x}=0,
$$

hence the results follows.

From Proposition 3.1, we get classical $L^{p}$ estimates, for any $1 \leq p \leq \infty$, on the distribution function $F$ : for all $t \in \mathbb{R}^{+}$

$$
\|F(t)\|_{L^{p}} \leq\left\|F_{0}\right\|_{L^{p}}
$$

and $F(t) \geq 0$ for any nonnegative initial data $F_{0}$.

Another remarkable property of our model is the preservation of the energy structure. Let us define the total energy $\mathcal{E}^{0}(t)$ : for a smooth solution $\left(F, \mathbf{E}_{F}\right)$ to (16),

$$
\mathcal{E}^{0}(t):=\int_{\Omega \times \mathbb{R}^{+} \times \mathbb{R}}\left(\frac{w^{2}+v_{\|}^{2}}{2}\right) F(t) w d w d v_{\|} d \mathbf{x}+\frac{1}{4 \pi} \int_{\Omega}\left|\mathbf{E}_{F}(t)\right|^{2} d \mathbf{x} .
$$


Proposition 3.2 Let $f_{\text {in }}^{\varepsilon}$ be a nonnegative function satisfying (10)-(11) and (13). Assume that the limiting system (16) has a smooth solution $\left(F, \mathbf{E}_{F}\right)$ and $\left(P, \mathbf{E}_{P}\right)$. Then, we have for any $t \geq 0$,

$$
\mathcal{E}^{0}(t)=\mathcal{E}^{0}(0)
$$

Proof Let us first multiply (20) by $v_{\|}^{2} / 2$ and integrate both in space and velocity, it gives

$$
\begin{aligned}
& \frac{d}{d t} \int \frac{\left|v_{\|}\right|^{2}}{2} F(t) w d w d v_{\|} d \mathbf{x}=-\int \frac{\partial \phi_{P}}{\partial x_{\|}} v_{\|} F(t) w d w d v_{\|} d \mathbf{x} \\
& \quad-\int \frac{\partial \phi_{F}}{\partial x_{\|}} v_{\|} P(t) w d w d v_{\|} d \mathbf{x} .
\end{aligned}
$$

Using the constraint in the parallel direction (16) on $F$, we obtain the following cancellation

$$
\begin{aligned}
\int \frac{\partial \phi_{P}}{\partial x_{\|}} v_{\|} F(t) w d w d v_{\|} d \mathbf{x} & =-\int \phi_{P} v_{\|} \frac{\partial F}{\partial x_{\|}}(t) w d w d v_{\|} d \mathbf{x} \\
& =\int \frac{\partial}{\partial v_{\|}}\left(\phi_{P} \frac{\partial \phi_{F}}{\partial x_{\|}} F(t)\right) w d w d v_{\|} d \mathbf{x}=0
\end{aligned}
$$

hence it yields

$$
\frac{d}{d t} \int \frac{\left|v_{\|}\right|^{2}}{2} F(t) w d w d v_{\|} d \mathbf{x}=-\int \frac{\partial \phi_{F}}{\partial x_{\|}} v_{\|} P(t) w d w d v_{\|} d \mathbf{x} .
$$

Then we multiply (20) by $w^{2} / 2$ and integrate both in space and velocity, we have

$$
\frac{d}{d t} \int \frac{w^{2}}{2} F(t) w d w d v_{\|} d \mathbf{x}=\int \nabla_{\mathbf{x}_{\perp}}^{\perp} b \cdot \nabla_{\mathbf{x}_{\perp}} \phi_{F} F \frac{w^{3}}{2 b^{2}} d w d v_{\|} d \mathbf{x} .
$$

Finally, we multiply (20) by the potential $\phi_{F}$ computed from $\rho_{F}$ and after integration, we have

$$
\begin{aligned}
\int \frac{\partial F}{\partial t} \phi_{F} w d w d v_{\|} d \mathbf{x}= & \int \nabla_{\mathbf{x}_{\perp} \phi_{F}} \mathbf{U}_{\perp} F w d w d v_{\|} d \mathbf{x}+\int \frac{\partial \phi_{F}}{\partial x_{\|}} v_{\|} P(t) w d w d v_{\|} d \mathbf{x} \\
= & -\int \nabla_{\mathbf{x}_{\perp}} \phi_{F} \cdot \nabla_{\mathbf{x}_{\perp}}^{\perp} b F \frac{w^{3}}{2 b^{2}} d w d v_{\|} d \mathbf{x} \\
& +\int \frac{\partial \phi_{F}}{\partial x_{\|}} v_{\|} P(t) w d w d v_{\|} d \mathbf{x} .
\end{aligned}
$$

On the other hand, by definition of $\rho_{F}$ and using the Poisson equation (18), we have

$$
\begin{aligned}
\int \frac{\partial F}{\partial t} \phi_{F} w d w d v_{\|} d \mathbf{x} & =\frac{1}{2 \pi} \int \frac{\partial \rho_{F}}{\partial t} \phi_{F} d \mathbf{x} \\
& =\frac{1}{4 \pi} \frac{d}{d t} \int\left|\nabla_{\mathbf{x}} \phi_{F}\right|^{2} d \mathbf{x} .
\end{aligned}
$$

Hence, gathering the later results, we obtain

$$
\begin{aligned}
\frac{1}{4 \pi} \frac{d}{d t} \int\left|\nabla_{\mathbf{x}} \phi_{F}\right|^{2} d \mathbf{x} & =-\int \nabla_{\mathbf{x}_{\perp}} \phi_{F} \cdot \nabla_{\mathbf{x}_{\perp}}^{\perp} b F \frac{w^{3}}{2 b^{2}} d w d v_{\|} d \mathbf{x} \\
& +\int \frac{\partial \phi_{F}}{\partial x_{\|}} v_{\|} P(t) w d w d v_{\|} d \mathbf{x} .
\end{aligned}
$$


Finally, adding (22), (23) and (24), we get the conservation of energy

$$
\frac{d}{d t} \mathcal{E}^{0}(t)=0
$$

\subsection{Invariance of the Magnetic Moment (First Adiabatic Invariant)}

In this section we assume that the external magnetic field does not depend on time. Then we define $\mu$ as the magnetic moment

$$
\mu=\frac{w^{2}}{2 b\left(\mathbf{x}_{\perp}\right)}
$$

and let us show that it is an invariant of the movement for the asymptotic model (16).

We compute the time derivative along the flow,

$$
\frac{d \mu}{d t}=\frac{w}{b} \frac{d w}{d t}-\frac{w^{2}}{2 b^{2}} \nabla_{\mathbf{x}_{\perp}} b \cdot \frac{d \mathbf{x}_{\perp}}{d t} .
$$

Using the characteristic curves to (16) and the orthogonality properties of the ${ }^{\perp}$ operator, it yields

$$
\begin{aligned}
\frac{d \mu}{d t} & =\frac{w^{2}}{2 b^{3}} \nabla_{\mathbf{x}_{\perp}}^{\perp} b \cdot \nabla_{\mathbf{x}_{\perp}} \phi_{F}+\frac{w^{2}}{2 b^{3}} \nabla_{\mathbf{x}_{\perp}} b \cdot\left(\nabla_{\mathbf{x}_{\perp}} \phi_{F}+\frac{w^{2}}{2} \frac{\nabla_{\mathbf{x}_{\perp}} b}{b}\right)^{\perp}, \\
& =\frac{w^{2}}{2 b^{3}} \nabla_{\mathbf{x}_{\perp}}^{\perp} b \cdot \nabla_{\mathbf{x}_{\perp}} \phi_{F}+\frac{w^{2}}{2 b^{3}} \nabla_{\mathbf{x}_{\perp}} b \cdot \nabla_{\mathbf{x}_{\perp}}^{\perp} \phi_{F}, \\
& =0 .
\end{aligned}
$$

Therefore, we can perform a change of variable on (16) to get the time evolution of the distribution function expressed in term of the magnetic moment $F \equiv F\left(t, \mathbf{x}, \mu, v_{\|}\right)$, it yields the following equation

$$
\left\{\begin{array}{l}
\frac{\partial F}{\partial t}+\mathbf{U}_{\perp} \cdot \nabla_{\mathbf{x}_{\perp}} F-\frac{\partial \phi_{P}}{\partial x_{\|}} \frac{\partial F}{\partial v_{\|}}+v_{\|} \frac{\partial P}{\partial x_{\|}}-\frac{\partial \phi_{F}}{\partial x_{\|}} \frac{\partial P}{\partial v_{\|}}=0 \\
v_{\|} \frac{\partial F}{\partial x_{\|}}-\frac{\partial \phi_{F}}{\partial x_{\|}} \frac{\partial F}{\partial v_{\|}}=0
\end{array}\right.
$$

where $\mathbf{U}_{\perp}$ is now given by

$$
\mathbf{U}_{\perp}=-\frac{1}{b}\left(\nabla_{\mathbf{x}_{\perp}} \phi_{F}+\mu \nabla_{\mathbf{x}_{\perp}} b\right)^{\perp}
$$

and the electric fields $\mathbf{E}_{F}, \mathbf{E}_{P}$ in (18). Notice that since $b\left(\mathbf{x}_{\perp}\right) d \mu d \mathbf{x}_{\perp}=w d w d \mathbf{x}_{\perp}$, the limiting system (25) can be written in conservative form when $b$ does not vary with time

$$
\left\{\begin{array}{l}
\frac{\partial b F}{\partial t}+\nabla_{\mathbf{x}_{\perp}} \cdot\left(\mathbf{U}_{\perp} b F\right)-\frac{\partial}{\partial v_{\|}}\left(\frac{\partial \phi_{P}}{\partial x_{\|}} b F\right)+\frac{\partial}{\partial x_{\|}}\left(v_{\|} b P\right)-\frac{\partial}{\partial v_{\|}}\left(\frac{\partial \phi_{F}}{\partial x_{\|}} b P\right)=0, \\
\frac{\partial}{\partial x_{\|}}\left(v_{\|} b F\right)-\frac{\partial}{\partial v_{\|}}\left(\frac{\partial \phi_{F}}{\partial x_{\|}} b F\right)=0 .
\end{array}\right.
$$




\subsection{Maxwell-Boltzmann Steady State}

In this part, we prove that the classical Maxwell-Boltzmann distribution is a steady state of the asymptotic model (16)

Proposition 3.3 Assume that the magnetic field b satisfies (5)-(6) and does not depend on time. Choosing $\left(P, \phi_{P}\right)=0$,

$$
F\left(\mathbf{x}, \mu, v_{\|}\right) \equiv \frac{1}{\sqrt{2 \pi}} \exp \left(-\left(\frac{v_{\|}^{2}}{2}+\phi_{F}(\mathbf{x})+\mu b\left(\mathbf{x}_{\perp}\right)\right)\right),
$$

where $\mu$ is the first adiabatic invariant, and $\phi_{F}$ solution to

$$
-\Delta \phi_{F}=e^{-\phi_{F}}-\rho_{0} .
$$

Then $\left(F, P, \phi_{F}, \phi_{P}\right)$ is a steady state solution to (16)-(18).

Proof Consider (16) written in the form of (25) with the first adiabatic invariant $\mu=w^{2} /(2 b)$. Therefore, the constraint in the parallel direction is automatically satisfied and also

$$
\nabla_{\mathbf{x}_{\perp}} F=-\left(\nabla_{\mathbf{x}_{\perp}} \phi+\mu \nabla_{\mathbf{x}_{\perp}} b\right) F,
$$

which is orthogonal to the velocity field $\mathbf{U}_{\perp}$, hence (25) is verified. Finally we compute

$$
\rho_{F}=\int_{\mathbb{R} \times \mathbb{R}^{+}} F\left(t, \mathbf{x}, \mu, v_{\|}\right) b\left(t, \mathbf{x}_{\perp}\right) d \mu d v_{\|}=\exp \left(-\phi_{F}\right),
$$

it yields the nonlinear Poisson equation (29).

\section{The Asymptotic Limit $\varepsilon \rightarrow 0$}

It is worth to mention here that these a priori estimates on the distribution function $F$ and the electric field $\mathbf{E}_{F}$ would give enough compactness to treat the nonlinear term $\mathbf{U}_{\perp} b F$, but we do not get any estimate on the additional term $\left(P, \mathbf{E}_{P}\right)$ so that the existence of weak solution to the limiting system (16) is still an open problem.

In order to establish a convergence result, we apply a formal analysis of the system (9). Applying a standard Hilbert expansion to $\left(f^{\mathcal{E}}, \mathbf{E}^{\varepsilon}\right)$ solution to the Vlasov-Poisson system (9), we get a hierarchy of differential equations which have to be solved at each order. Here, we take advantage of the simple structure of the magnetic field to solve explicitly each problem and get the asymptotic model (16).

\subsection{The Hilbert Expansion}

Since the leading order term in (9) involves the effect of a circular motion around the magnetic field lines, we now specifically examine the properties of this operator. Let us denote by $\mathcal{L}$ the following operator

$$
\mathcal{L} f=-b\left(t, \mathbf{x}_{\perp}\right) \mathbf{v}^{\perp} \cdot \nabla_{\mathbf{v}} f .
$$

We have the following result 
Lemma 4.1 Assume that $b$ satisfies (5)-(6). Then, the null space $\operatorname{ker} \mathcal{L}$ of $\mathcal{L}$ consists of functions which only depend on the parallel component $v_{\|}$and on the amplitude of $\mathbf{v}_{\perp}$, that is, $w=\left|\mathbf{v}_{\perp}\right|$,

$$
\mathcal{L} f=0 \Longleftrightarrow f(\mathbf{v}) \equiv \tilde{f}\left(w, v_{\|}\right) \text {with }\left(w, v_{\|}\right) \in \mathbb{R}^{+} \times \mathbb{R} .
$$

Proof On the one hand, we notice that the magnetic field $\mathbf{B}_{\text {ext }}$ only acts on $\mathbf{v}_{\perp}=\left(v_{x}, v_{y}\right)$, which means that

$$
\mathcal{L} f=-b\left(t, \mathbf{x}_{\perp}\right) \mathbf{v}_{\perp}^{\perp} \cdot \nabla_{\mathbf{v}_{\perp}} f,
$$

where now ${ }^{\perp}$ acts on a vector of $\mathbb{R}^{2}$ and $\mathbf{v}_{\perp}^{\perp}=\left(v_{y},-v_{x}\right)$. Then, applying a change of variable to polar coordinates on $\mathbf{v}_{\perp} \in \mathbb{R}^{2}$, it yields

$$
\mathcal{L} f=b\left(t, \mathbf{x}_{\perp}\right) \frac{\partial f}{\partial \theta} .
$$

From (6), the magnetic field does not vanish, hence we get $\mathcal{L} f=0$ if and only if $f(\mathbf{v}) \equiv$ $\tilde{f}\left(w, v_{\|}\right)$, which proves (30).

Now, our goal is to find the asymptotic limit $\varepsilon \rightarrow 0$ to the Vlasov-Poisson system (9). We start by assuming that $\left(f^{\varepsilon}, \mathbf{E}^{\varepsilon}\right)$ admits an Hilbert expansion:

$$
f^{\varepsilon}=f_{0}+\varepsilon f_{1}+\varepsilon^{2} f_{2}+\ldots,
$$

and

$$
\mathbf{E}^{\varepsilon}=\mathbf{E}_{0}+\varepsilon \mathbf{E}_{1}+\varepsilon^{2} \mathbf{E}_{2}+\ldots
$$

Inserting these expansions in the Vlasov-Poisson system (9), we find for the leading order $\varepsilon^{-2}$,

$$
\mathcal{L} f_{0}=0,
$$

the order $\varepsilon^{-1}$

$$
\mathcal{L} f_{1}=\mathbf{v} \cdot \nabla_{\mathbf{x}} f_{0}+\mathbf{E}_{0} \cdot \nabla_{\mathbf{x}} f_{0}
$$

and the order $\varepsilon^{0}$

$$
\mathcal{L} f_{2}=\frac{\partial f_{0}}{\partial t}+\mathbf{v} \cdot \nabla_{\mathbf{x}} f_{1}+\mathbf{E}_{0} \cdot \nabla_{\mathbf{v}} f_{1}+\mathbf{E}_{1} \cdot \nabla_{\mathbf{v}} f_{0}
$$

In order to solve eq. $\mathcal{L} f=h$, we proceed in two steps :

- we verify the solvability condition $\Pi h=0$;

- we compute $f$ by integrating $h$ over $\theta$.

\subsection{Proof of Theorem 2.2}

In this section we derive an asymptotic model for the limit $f_{0}$ of $f^{\varepsilon}$ by formally passing to the limit $\varepsilon \rightarrow 0$ in the Vlasov-Poisson system (9). This model will be deduced by solving the sequence of equations appearing in the Hilbert expansion (31)-(33).

First, by a simple application of Lemma 4.1, the leading order of the Hilbert expansion (31) can be directly solved. The function $f_{0}$ does not depend on $\theta \in[0,2 \pi]$ and $f_{0} \equiv$ $F\left(t, \mathbf{x}, w, v_{\|}\right)$for any $\mathbf{v}=\left(\mathbf{v}_{\perp}, v_{\|}\right) \in \mathbb{R}^{3}$ and at time $t=0$, we set $F(0)=\Pi f_{\text {in, } 0}$, where $f_{\text {in }, 0}$ is given from the expansion of the initial data $f_{\text {in }}^{\mathcal{E}}$ in (13). 
Moreover, substituting the Hilbert expansion to $\mathbf{E}^{\varepsilon}$ in the Poisson equation in (9), gives that $\mathbf{E}_{0}=\mathbf{E}_{F}:=-\nabla \phi_{F}$ with

$$
\Delta \phi_{F}=\rho_{F}=2 \pi \int_{\mathbb{R}^{+} \times \mathbb{R}} F w d w d v_{\|} .
$$

Now, the goal is to find the equation satisfied by $F$, hence we turn to (32) and first set

$$
\mathbf{G}\left(t, \mathbf{x}, w, v_{\|}\right):=w \nabla_{\mathbf{x}_{\perp}} F-\nabla_{\mathbf{x}_{\perp}} \phi_{F} \frac{\partial F}{\partial w} .
$$

Then we prove the following Proposition.

Proposition 4.2 Assume that b satisfies (5)-(6) and consider $\left(F, \mathbf{E}_{F}\right)$ the leading order of the Hilbert expansion (12). Then Eq. (32) admits a solution $f_{1}$ if and only if $F$ satisfies the solvability condition

$$
v_{\|} \frac{\partial F}{\partial x_{\|}}-\frac{\partial \phi_{F}}{\partial x_{\|}} \frac{\partial F}{\partial v_{\|}}=0 .
$$

Moreover, if this condition is satisfied, then there exists a function $P \in \operatorname{ker} \mathcal{L}$ such that

$$
\left\{\begin{array}{l}
f_{1}(t, \mathbf{x}, \mathbf{v})=-\frac{1}{b\left(t, \mathbf{x}_{\perp}\right)} \mathbf{e}_{\theta} \cdot \mathbf{G}\left(t, \mathbf{x}, w, v_{\|}\right)+P\left(t, \mathbf{x}, w, v_{\|}\right), \\
\mathbf{E}_{1}(t, \mathbf{x})=\mathbf{E}_{P}:=-\nabla \phi_{P},
\end{array}\right.
$$

with $\mathbf{e}_{\theta}=(-\sin \theta, \cos \theta)^{t}, \phi_{P}$ satisfies the Poisson equation (18) and at time $t=0$, we have $P(0)=\Pi f_{1}(0, \mathbf{x}, \mathbf{v})$.

Proof Thanks to the definition of $\mathbf{G}$, we write (32) as

$$
\mathcal{L} f_{1}=\mathbf{e}_{w} \cdot \mathbf{G}\left(t, \mathbf{x}, w, v_{\|}\right)+v_{\|} \frac{\partial F}{\partial x_{\|}}-\frac{\partial \phi_{F}}{\partial x_{\|}} \frac{\partial F}{\partial v_{\|}} .
$$

On the one hand, we require that the solvability condition of (32) is satisfied

$$
\Pi \mathcal{L} f_{1}=0 \text {. }
$$

Since $\Pi \mathbf{e}_{w}=0$, a necessary and sufficient condition for the solvability of (32) is that $F$ satisfies the following condition

$$
v_{\|} \frac{\partial F}{\partial x_{\|}}-\frac{\partial \phi_{F}}{\partial x_{\|}} \frac{\partial F}{\partial v_{\|}}=0,
$$

which corresponds to the constraint equation in (16).

On the other hand, assuming that this solvability condition is verified, we can explicitly solve (32) by integration with respect to $\theta \in[0,2 \pi]$. Then there exists a function $P \in \operatorname{ker} \mathcal{L}$ such that for any $(t, \mathbf{x}, \mathbf{v}) \in \mathbb{R}^{+} \times \mathbb{R}^{3} \times \mathbb{R}^{3}$,

$$
f_{1}(t, \mathbf{x}, \mathbf{v})=-\frac{1}{b\left(t, \mathbf{x}_{\perp}\right)} \mathbf{e}_{\theta} \cdot \mathbf{G}\left(t, \mathbf{x}, w, v_{\|}\right)+P\left(t, \mathbf{x}, w, v_{\|}\right),
$$

where $\Pi \mathbf{e}_{\theta}=0$ and from the initial condition (13), it gives that

$$
P(0)=\Pi f_{\text {in }, 1} .
$$

Finally, substituting the Hilbert expansion to $\mathbf{E}^{\varepsilon}$ in the Poisson equation in (9) and using that

$$
\Pi f_{1}=\frac{1}{b} \Pi \mathbf{e}_{\theta} \cdot \mathbf{G}+\Pi P=\Pi P,
$$


we observe that

$$
\rho_{1}=\int_{\mathbb{R}^{3}} f_{1} d \mathbf{v}=2 \pi \int_{\mathbb{R}^{+} \times \mathbb{R}} P w d w d v_{\|},
$$

which gives that $\mathbf{E}_{1}=\mathbf{E}_{P}:=-\nabla \phi_{P}$ with $\phi_{P}$ solution to the Poisson equation

$$
-\Delta \phi_{P}=\rho_{F}=2 \pi \int_{\mathbb{R}^{+} \times \mathbb{R}} P w d w d v_{\|}
$$

Note that $f_{1}$ depends now on the whole variable $(\mathbf{x}, \mathbf{v}) \in \mathbb{R}^{3} \times \mathbb{R}^{3}$. Finally, the equation satisfied by $F$ now appears as the solvability condition of (33).

Proposition 4.3 Assume that b satisfies (5)-(6) and consider $\left(F, \mathbf{E}_{F}\right)$ the leading order of the Hilbert expansion (12). Then, equation (33) admits a solution $f_{2}$ if and only if $F$ satisfies the first equation in (16), that is,

$$
\frac{\partial F}{\partial t}+\mathbf{U}_{\perp} \cdot \nabla_{\mathbf{x}_{\perp}} F+u_{w} \frac{\partial F}{\partial w}-\frac{\partial \phi_{P}}{\partial x_{\|}} \frac{\partial F}{\partial v_{\|}}+v_{\|} \frac{\partial P}{\partial x_{\|}}-\frac{\partial \phi_{F}}{\partial x_{\|}} \frac{\partial P}{\partial v_{\|}}=0,
$$

where the drift velocity $\mathbf{U}_{\perp}$ and $u_{w}$ are given by (17).

Proof As before we apply the solvability condition to (33), which corresponds to

$$
\Pi \mathcal{L} f_{2}=0,
$$

or it can be written as

$$
\int_{0}^{2 \pi}\left(\frac{\partial F}{\partial t}+\mathbf{v} \cdot \nabla_{\mathbf{x}} f_{1}+\mathbf{E}_{F} \cdot \nabla_{\mathbf{v}} f_{1}+\mathbf{E}_{P} \cdot \nabla_{\mathbf{v}} F\right) d \theta=0,
$$

where $\mathbf{E}_{Q}=-\nabla \phi_{Q}$ corresponds to electric field obtained by solving the Poisson equation (18).

Let us compute explicitly each term with respect to $\left(F, \mathbf{E}_{F}\right)$ and $\left(P, \mathbf{E}_{P}\right)$ given from the previous analysis.

On the one hand, since the distribution function $F$ does not depend on the angular velocity, we have that

$$
\frac{1}{2 \pi} \int_{0}^{2 \pi} \frac{\partial F}{\partial t} d \theta=\frac{\partial F}{\partial t}
$$

and

$$
\frac{1}{2 \pi} \int_{0}^{2 \pi} \mathbf{E}_{P} \cdot \nabla_{\mathbf{v}} F d \theta=-\frac{\partial \phi_{P}}{\partial x_{\|}} \frac{\partial F}{\partial v_{\|}} .
$$

On the other hand, from the definition of $f_{1}$ given in Proposition 4.2, we get that

$$
\frac{1}{2 \pi} \nabla_{\mathbf{x}} \cdot\left(\int_{0}^{2 \pi} v f_{1} d \theta\right)=-\frac{w}{2} \nabla_{\mathbf{x}_{\perp}} \cdot\left(\frac{\mathbf{G}^{\perp}}{b}\right)+v_{\|} \frac{\partial P}{\partial x_{\|}},
$$

with

$$
\mathbf{G}^{\perp}=w \nabla_{\mathbf{x}_{\perp}}^{\perp} F-\nabla_{\mathbf{x}_{\perp}}^{\perp} \phi_{F} \frac{\partial F}{\partial w} .
$$


Then, since the operator $\nabla_{\mathbf{x}_{\perp}} \cdot \nabla_{\mathbf{x}_{\perp}}^{\perp}=0$, it yields that

$$
\frac{1}{2 \pi} \nabla_{\mathbf{x}} \cdot\left(\int_{0}^{2 \pi} v f_{1} d \theta\right)=\frac{w}{2 b} \nabla_{\mathbf{x}_{\perp}}^{\perp} \phi_{F} \frac{\partial}{\partial w}\left(\nabla_{\mathbf{x}_{\perp}} F\right)+\frac{w}{2} \frac{\nabla_{\mathbf{x}_{\perp}} b}{b^{2}} \cdot \mathbf{G}^{\perp}+v_{\|} \frac{\partial P}{\partial x_{\|}} .
$$

Finally, we evaluate the penultimate term in (35). From the expression of $f_{1}$ in Proposition 4.2, we obtain

$$
\frac{1}{2 \pi} \mathbf{E}_{F} \cdot\left(\int_{0}^{2 \pi} \nabla_{\mathbf{v}} f_{1} d \theta\right)=\frac{1}{2 b} \nabla_{\mathbf{x}_{\perp}} \phi_{F} \cdot\left(\frac{\mathbf{G}}{w}+\frac{\partial \mathbf{G}}{\partial w}\right)^{\perp}-\frac{\partial \phi_{F}}{\partial x_{\|}} \frac{\partial P}{\partial v_{\|}} .
$$

Hence using the orthogonality property and $\mathbf{u}^{\perp} \cdot \mathbf{w}=-\mathbf{w}^{\perp} \cdot \mathbf{u}$ for any $(\mathbf{u}, \mathbf{w}) \in \mathbb{R}^{2} \times \mathbb{R}^{2}$, it yields

$$
\frac{1}{2 \pi} \mathbf{E}_{F} \cdot\left(\int_{0}^{2 \pi} \nabla_{\mathbf{v}} f_{1} d \theta\right)=-\frac{\nabla_{\mathbf{x}_{\perp}}^{\perp} \phi_{F}}{b} \cdot \nabla_{\mathbf{x}_{\perp}} F+\frac{w}{2 b} \nabla_{\mathbf{x}_{\perp}} \phi_{F} \cdot \frac{\partial}{\partial w}\left(\nabla_{\mathbf{x}_{\perp}}^{\perp} F\right)-\frac{\partial \phi_{F}}{\partial x_{\|}} \frac{\partial P}{\partial v_{\|}} .
$$

Gathering (36) and (37), we get some cancellation and it yields to the following expression

$$
\begin{aligned}
\frac{1}{2 \pi} \int_{0}^{2 \pi}\left(\nabla_{\mathbf{x}} \cdot v f_{1}+\mathbf{E}_{F} \cdot \nabla_{\mathbf{v}} f_{1}\right) d \theta & =\mathbf{U}_{\perp} \cdot \nabla_{\mathbf{x}_{\perp}} F+u_{w} \frac{\partial F}{\partial w} \\
& +v_{\|} \frac{\partial P}{\partial x_{\|}}-\frac{\partial \phi_{F}}{\partial x_{\|}} \frac{\partial P}{\partial v_{\|}} .
\end{aligned}
$$

where

$$
\mathbf{U}_{\perp}=-\frac{1}{b}\left(\nabla_{\mathbf{x}_{\perp}} \phi+\frac{w^{2}}{2 b} \nabla_{\mathbf{x}_{\perp}} b\right)^{\perp}, \quad u_{w}=\frac{w}{2 b^{2}} \nabla_{\mathbf{x}_{\perp}}^{\perp} b \cdot \nabla_{x_{\perp}} \phi_{F} .
$$

Finally, the solvability condition on $f_{2}$ is satisfied once the distribution function $F$ is solution to the following equation

$$
\frac{\partial F}{\partial t}+\mathbf{U}_{\perp} \cdot \nabla_{\mathbf{x}_{\perp}} F+u_{w} \frac{\partial F}{\partial w}-\frac{\partial \phi_{P}}{\partial x_{\|}} \frac{\partial F}{\partial v_{\|}}+v_{\|} \frac{\partial P}{\partial x_{\|}}-\frac{\partial \phi_{F}}{\partial x_{\|}} \frac{\partial P}{\partial v_{\|}}=0,
$$

which completes the first part of the proof.

When the solvability condition is satisfied, then the equation (33), can be solved explicitly and the solution $f_{2}$ only depends on $\left(F, \mathbf{E}_{F}\right)$ and $\left(P, \mathbf{E}_{P}\right)$ and a function $R \in \operatorname{ker} \mathcal{L}$.

\section{Open Problems and Conclusion}

In this paper we studied the long time behavior of the solution to the Vlasov-Poisson system (9) with a strong external magnetic field $\mathbf{B}_{\text {ext }}=(0,0, b)$. We provide a formal analysis based on a Hilbert type expansion of the solution and the formal limit is solution to a reduced kinetic model (16) where the solution does not depend anymore on the angular perpendicular velocity $\theta \in(0,2 \pi)$. As fas as we know, this reduced model is new and satisfies some fundamental properties as the correct drift velocities $\mathbf{E} \times \mathbf{B}_{\text {ext }}$, gradient B-drift, conservation of energy, entropy, and invariance of the magnetic moment. However due to the constraint property in the parallel direction in (16), it deserves more attention in term of well-posedness, regularity of solutions and numerical discretization. 
About the generalization to an arbitrary external magnetic field Here we focus on the formal analysis when the magnetic field only applies in the $z$-direction. We may also consider an arbitrary external magnetic field $\mathbf{B}_{\text {ext }}$ in order to get a more elaborated limiting system taking into account curvature drift, polarization effects, etc. This can be done by following the guideline of the analysis performed in [5].

The main modification is that we cannot anymore work in Cartesian coordinate but need to apply a change of coordinates to follow the parallel direction of the external magnetic field

$$
\mathbf{v}=v_{\|} \mathbf{b}(t, \mathbf{x})+\mathbf{v}_{\perp}
$$

where $\mathbf{b}$ represents a unit vector parallel to the magnetic field. This moving frame induces new drift velocity both in the parallel and perpendicular direction to the magnetic field which have to be taken into account.

About the rigorous justification of the limiting system To justify our asymptotic analysis, we should consider a smooth solution to the Vlasov-Poisson system (9), and should assume that the limiting system also admits a smooth solution : for any $k \geq 0,\left(F, \mathbf{E}_{F}\right) \in \mathcal{C}_{c}^{k+3} \times \mathcal{C}^{k+3}$ and $\left(P, \mathbf{E}_{P}\right) \in \mathcal{C}_{c}^{k+2} \times \mathcal{C}^{k+2}$, where $\mathcal{C}^{k}$ is the space of functions with $k$ continuous derivatives and $\mathcal{C}_{c}^{k}$ the sub-space of $\mathcal{C}^{k}$ with compactly supported functions.

Then, we construct $\left(F^{\varepsilon}, \mathbf{E}^{\varepsilon}\right)$ by

$$
F^{\varepsilon}=F+\varepsilon f_{1}+\varepsilon^{2} f_{2}, \quad \mathbf{E}^{\varepsilon}=\mathbf{E}_{F}+\varepsilon \mathbf{E}_{P},
$$

where $f_{1}$ and $f_{2}$ are solutions to (32) and (33), and $f_{2}$ such that $\Pi f_{2}=0$. Therefore $\left(F^{\varepsilon}, \mathbf{E}^{\varepsilon}\right) \in \mathcal{C}_{c}^{k+1} \times \mathcal{C}^{k+1}$ satisfies the Vlasov-Poisson system (9) with a source term $\left(R^{\varepsilon}\right)_{\varepsilon>0}$

$$
\left\{\begin{array}{l}
\varepsilon \frac{\partial F^{\varepsilon}}{\partial t}+\mathbf{v} \cdot \nabla_{\mathbf{x}} F^{\varepsilon}+\left(\mathbf{E}^{\varepsilon}+\frac{b}{\varepsilon} \mathbf{v}^{\perp}\right) \cdot \nabla_{\mathbf{v}} F^{\varepsilon}=-\varepsilon R^{\varepsilon}, \\
-\Delta \phi^{\varepsilon}=\rho^{\varepsilon}=\int_{\mathbb{R}^{3}} F^{\varepsilon} d \mathbf{v}, \quad \mathbf{E}^{\varepsilon}=-\nabla_{\mathbf{x}} \phi^{\varepsilon}, \\
F^{\varepsilon}(0)=F(0)+\varepsilon f_{1}(0)+\varepsilon^{2} f_{2}(0),
\end{array}\right.
$$

with

$$
R^{\varepsilon}=\varepsilon\left(\frac{\partial f_{1}}{\partial t}+\mathbf{E}_{P} \cdot \nabla_{\mathbf{v}} f_{1}\right)+\varepsilon^{2}\left(\frac{\partial f_{2}}{\partial t}++\mathbf{v} \cdot \nabla_{\mathbf{x}} f_{2}+\mathbf{E}^{\varepsilon} \cdot \nabla_{\mathbf{v}} f_{2}\right)
$$

such that for all $k \geq 0$,

$$
\left\|R^{\varepsilon}\right\|_{H^{k}} \leq C\left[\varepsilon\left(\|F\|_{H^{k+2}}+\left\|\mathbf{E}_{F}\right\|_{H^{k+2}}\right)+\varepsilon^{2}\left(\|F\|_{H^{k+3}}+\left\|\mathbf{E}_{F}\right\|_{H^{k+3}}\right)\right] .
$$

The second step is to establish a comparison principle on the Vlasov-Poisson system (9) to prove the convergence

$$
\left\|F^{\varepsilon}-f^{\varepsilon}\right\|_{H^{k}} \leq C\left\|R^{\varepsilon}\right\|_{H^{k}} .
$$

About existence, uniqueness and regularity of solutions to the limiting system (16) From the $L^{p}$ estimate on $F$ and the regularizing properties of the Poisson equation, we get enough compactness to treat the nonlinear term $\mathbf{U}_{\perp} F$. However, the lack of estimates on the Lagrange multiplier $\left(P, \mathbf{E}_{P}\right)$ is the main issue to prove existence of weak solutions. Unfortunately, the constraint introduced in the limiting system (16) is not standard in kinetic theory and fluid 
mechanics since the constraint is nonlinear in the sense that the differential operator $\mathcal{T}_{F}$ applied to $P$ depends on the solution itself via the potential $\phi_{F}$

$$
\mathcal{T}_{F} P:=v_{\|} \frac{\partial P}{\partial x_{\|}}-\frac{\partial \phi_{F}}{\partial x_{\|}} \frac{\partial P}{\partial v_{\|}} .
$$

Therefore, we cannot simply eliminate the constraint by introducing an appropriate functional space. However for some situations, it is possible to construct solutions. We have already mentioned that the Maxwell-Boltzmann distribution (28) is a steady state for (16). Another example is when $\left(P, \phi_{P}\right)=0$ and $F$ does not depend on $x_{\|}$. We choose $F \equiv \mathcal{G}\left(t, \mathbf{x}_{\perp}\right) \mathcal{M}\left(\mu, v_{\|}\right)$, where $\mathcal{G}$ is solution to the guiding center equation

$$
\frac{\partial \mathcal{G}}{\partial t}+\mathbf{U}_{\perp} \cdot \nabla_{\mathbf{x}_{\perp}} \mathcal{G}=0
$$

with $\mathbf{U}_{\perp}$ is given by (26) and $\mathcal{M}$ is an arbitrary smooth and nonnegative function. The constraint in (16) is automatically satisfied and $\left(F, \mathbf{E}_{F}\right)$ is solution to (16).

About the long time behavior of the solution to the limiting system (16) The constraint in the parallel direction to the magnetic field

$$
v_{\|} \frac{\partial F}{\partial x_{\|}}-\frac{\partial \phi_{F}}{\partial x_{\|}} \frac{\partial F}{\partial v_{\|}}=0,
$$

is very unusual and may be very strong. Therefore, it is not guaranteed that the limiting system (16) can describe accurately plasma turbulence from current and spatial gradients and a stability analysis of the particular solutions (28) may be investigated.

About the numerical simulation of (16) Finally the numerical approximation of the limiting system (16) should be investigated to study the relevance of such a model. This system has a clear advantage from a numerical point of view since the stiffness due to the external magnetic field of the Vlasov-Poisson system (9) has been removed and the fast variable $\theta \in(0,2 \pi)$ is eliminated by averaging. However, the discretization of (16) is not straightforward due to the constraint in the parallel direction to the magnetic field and a specific investigation have to be done. One possibility is to follow the strategy applied in fluid mechanics for the two dimensional incompressible Euler system using Galerkin discontinuous methods [38].

Acknowledgments Both authors wish to express their gratitude to Eric Sonnendruücker for fruitful discussions on this topic. This work has been supported by the Engineering and Physical Sciences Research Council (EPSRC) under Grant Ref. EP/M006883/1, and by the National Science Foundation (NSF) under grant RNMS11-07444 (KI-Net). This work has also been carried out within the framework of the EUROfusion Consortium and has received funding from the Euratom research and training programme 2014-2018 under Grant Agreement No. 633053. The views and opinions expressed herein do not necessarily reflect those of the European Commission. F. F. is grateful to the Department of Mathematics of Imperial College of London for its hospitality. P. D. is on leave from CNRS, Institut de Mathématiques, Toulouse, France. He acknowledges support from the Royal Society and the Wolfson foundation through a Royal Society Wolfson Research Merit Award.

Open Access This article is distributed under the terms of the Creative Commons Attribution 4.0 International License (http://creativecommons.org/licenses/by/4.0/), which permits unrestricted use, distribution, and reproduction in any medium, provided you give appropriate credit to the original author(s) and the source, provide a link to the Creative Commons license, and indicate if changes were made. 


\section{References}

1. Antonsen, T.M., Lane, B.: Kinetic equations for low frequency instabilities in inhomogeneous plasmas. Phys. Fluids 23, 1205-1214 (1980)

2. Arsen'ev, A.A.: Global existence of a weak solution of Vlasov's system of equations. Zh. vychisl. Mat. i. Mat. Fiz. 15, 136-147 (1975)

3. Bellan, P.M.: Fundamentals of Plasma Physics. Cambridge University Press, Cambridge (2006)

4. Cheverry, Ch.: Can one hear whistler waves? Commun. Math. Phys. 338(2), 641-703 (2015)

5. Degond, P., Hirtsoaga, S., Vignal, M.-H.: The Vlasov model under large magnetic fields in the low-Mach number regime. preprint arXiv:0905.2400 (2009)

6. DiPerna, R.J., Lions, P.-L.: Solutions globales d'équations du type Vlasov-Poisson. C. R. Acad. Sci. Paris Sér. I Math. 307, 306-329 (1988)

7. Beer, M.A., Hammett, G.W.: Toroidal Gyrofluid Equations for Simulations of Tokamak Turbulence. Phys. Plasmas 3, 4046-4064 (1996)

8. Bostan, M.: The Vlasov-Maxwell system with strong initial magnetic field: guiding-center approximation. Multiscale Model. Simul. 6(3), 1026-1058 (2007)

9. Bostan, M.: The Vlasov-Poisson system with strong external magnetic field. Finite Larmor radius regime. Asymptot. Anal. 61, 91-123 (2009)

10. Bostan, M.: Gyrokinetic Vlasov equation in three dimensional setting. Second order approximation. Multiscale Model. Simul. 8(5), 1923-1957 (2010)

11. Bostan, M.: Gyrokinetic models for strongly magnetized plasmas with general magnetic shape. Discret. Contin. Dyn. Syst. Ser. S 5(2), 257-269 (2012)

12. Bottino, A., Tran, T.M., Sauter, O., Vaclavik, J., Villard, L.: Linear gyrokinetic simulations using particles for small perpendicular wavelength perturbations. In: Theory of Fusion Plasmas, Proceedings of the International Workshop, Varenna, 2000, Compositori, Bologna, pp. 327-332 (2001)

13. Brenier, Y.: Convergence of the Vlasov-Poisson system to the incompressible Euler equations. Commun. Partial Differ. Equ. 25, 737-754 (2000)

14. Brizard, A.J., Hahm, T.S.: Foundations of nonlinear gyrokinetic theory. Rev. Modern Phys. 79, 421-468 (2007)

15. Dimits, A.M., et al.: Comparisons and physics basis of tokamak transport models and turbulence simulations. Phys. Plasmas 7, 969-983 (2000)

16. Dorland, W., Hammett, G.W.: Gyrofluid turbulence models with kinetic effects. Phys. Fluids B Plasmas 5, 812-835 (1993)

17. Falchetto, G.L., Ottaviani, M.: Effect of collisional zonal-flow damping on flux-driven turbulent transport. Phys. Rev. Lett. 92, 025002 (2004)

18. Frénod, E., Sonnendrücker, E.: Homogenization of the Vlasov equation and of the Vlasov-Poisson system with a strong external magnetic field. Asymptot. Anal. 18, 193-213 (1998)

19. Frénod, E., Sonnendrücker, E.: Long time behavior of the two-dimensional Vlasov equation with a strong external magnetic field. Math. Models Methods Appl. Sci. 10, 539-553 (2000)

20. Frénod, E., Raviart, P.-A., Sonnendrücker, E.: Two-scale expansion of a singularly perturbed convection equation. J. Math. Pures Appl. 80, 815-843 (2001)

21. Garbet, X., Bourdelle, C., Hoang, G.T., Maget, P., Benkadda, S., Beyer, P., Figarella, C., Voitsekovitch, I., Agullo, O., Bian, N.: Global simulations of ion turbulence with magnetic shear reversal. Phys. Plasmas 8, 2793-2803 (2001)

22. Ghendrih, P., Hauray, M., Nouri, A.: Derivation of a gyrokinetic model. Existence and uniqueness of specific stationary solution. Kinet. Relat. Models 2(4), 707-725 (2009)

23. Golse, F., Saint-Raymond, L.: The Vlasov-Poisson system with strong magnetic field. J. Math. Pures Appl. 78, 791-817 (1999)

24. Golse, F., Saint-Raymond, L.: The Vlasov-Poisson system with strong magnetic field in quasineutral regime. Math. Models Methods Appl. Sci. 13(5), 661-714 (2003)

25. Hammett, G.W., Beer, M.A., Dorland, W., Cowley, S.C., Smith, S.A.: Developments in the gyrofluid approach to tokamak turbulence simulations. Plasma Phys. Control. F 35, 973-985 (1993)

26. Han-Kwan, D.: Effect of the polarization drift in a strongly magnetized plasma. ESAIM Math. Model. Numer. Anal. 46, 929-947 (2012)

27. Han-Kwan, D.: The three-dimensional finite Larmor radius approximation. Asymptot. Anal. 66(1), 9-33 (2010)

28. Han-Kwan, D.: On the confinement of a tokamak plasma. SIAM J. Math. Anal. 42(6), 2337-2367 (2010)

29. Han-Kwan, D.: On the three-dimensional finite Larmor radius approximation: the case of electrons in a fixed background of ions. Ann. Inst. H. Poincaré Anal. Non Linéaire 30(6), 1127-1157 (2013) 
30. Hauray, M., Nouri, A.: Well-posedness of a diffusive gyro-kinetic model. Ann. Inst. H. Poincaré Anal. Non Linéaire 28, 529-550 (2011)

31. Hazeltine, R.D., Ware, A.A.: The drift kinetic equation for toroidal plasmas with large mass velocities. Plasma Phys. 20, 673-678 (1978)

32. Hazeltine, R.D., Meiss, J.D.: Plasma Confin. Dover Publications, Mineola (2003)

33. Idomura, Y., Tokuda, S., Kishimoto, Y.: Global gyrokinetic simulation of ion temperature gradient driven turbulence in plasmas using a canonical Maxwellian distribution. Nucl. Fusion 43, 234-243 (2003)

34. Kim, C.C., Parker, S.E.: Massively parallel three dimensional toroidal gyrokinetic flux-tube turbulence simulations. J. Comput. Phys. 161, 589-604 (2000)

35. Lee, W.W.: Gyrokinetic approach in particle simulation. Phys. Fluids 26, 556-562 (1983)

36. Lifshitz, E.M., Pitaevskii, L.P.: Physical Kinetics, Course in Theoretical Physics, vol. 10. Butterworthheinemann, Oxford (2006)

37. Lin, Z., Hahm, T.S., Lee, W.W., Tang, W.M., White, R.B.: Gyrokinetic simulations in general geometry and applications to collisional damping of zonal flows. Phys. Plasmas 7(5), 1857-1862 (2000)

38. Liu, J.G., Shu, C.-W.: A high-order discontinuous Galerkin method for 2D incompressible flows. J. Comput. Phys. 160(2), 577-596 (2000)

39. Miyamoto, K.: Plasma Physics and Controlled Nuclear Fusion, vol. 38. Springer, Berlin (2006)

40. Naulin, V.: Electromagnetic transport components and sheared flows in drift-Alfvèn turbulence. Phys. Plasmas 10, 4016-4028 (2003)

41. Ottaviani, M., Manfredi, G.: The gyro-radius scaling of ion thermal transport from global numerical simulations of ion temperature gradient driven turbulence. Phys. Plasmas 6, 3267-3275 (1999)

42. Saint-Raymond, L.: Control of large velocities in the two-dimensional gyrokinetic approximation. J. Math. Pures Appl. 81(4), 379-399 (2002)

43. Scott, B.D.: Free-energy conservation in local gyrofluid models. Phys. Plasmas 12, 1102307 (2005)

44. Sugama, H., Watanabe, T.H., Horton, W.: Comparison between kinetic and fluid simulations of slab ion temperature gradient driven turbulence. Phys. Plasmas 10, 726-736 (2003)

45. Tran, T.M., Appert, K., Fivaz, M., Jost, G., Vaclavik, J., Villard, L.: Global gyrokinetic simulation of IonTemperature-Gradient driven instabilities. In: Theory of Fusion Plasmas, Proceedings of the International Workshop, Varenna, 1998, Compositori, Bologna, pp. 45-49 (1999)

46. Xu, X.Q., Cohen, R.H., Rognlien, T.D., Myra, J.R.: Low-to-high confinement transition simulations in divertor geometry. Phys. Plasmas 7, 1951-1958 (2000) 